\title{
Orientation effects on contour interaction in the Zöllner illusion
}

\author{
K. GEOFFREY WHITE \\ Colorado College, Colorado Springs, Colorado 80903
}

\begin{abstract}
The magnitude of the Zöllner illusion was measured as a joint function of the angle of intersection between inducing and test contours and the orientation of the complete display. The intersect angle at which judgmental error was maximal varied as the display was rotated from 0 to 90 deg. An explanation of the Zöllner illusion in terms of selective adaptation of neural orientation specific detectors accounts for the interactive effects of display orientation and intersect angle if it is assumed that different orientation detectors have different tuning characteristics.
\end{abstract}

One class of geometrical illusions, the distortion illusions (Robinson, 1972), is characterized by distortion of the angles between intersecting test and inducing contours. At a descriptive level, the acute angle between the contours of configurations such as the Zölner, Poggendorti, Orbison, Wundt, and Hering illusions is perceptually enlarged, although in the case of the Poggendorff figure it seems to be the obtuse angle between the diagonal and parallel lines which is the important factor in the generation of the illusion (Daly, 1973; Krantz \& Weintraub, 1973). An alternative, although equivalent, description is that the acute angles in the figure exhibit "regression towards right angles" (Helmholtz, 1866; Hering, 1861). The angular distortion might in fact be a combination of a contrast effect between the intersecting contours and a normalization effect whereby the contours are attracted towards a spatial (horizontal-vertical) norm.

Acute-angle enlargement has been invoked by a number of investigators as the major principle governing the distortion illusions (Békésy, 1967; Berliner \& Berliner, 1948; Day, 1965; Piaget. 1969; Wallace, 1966. 1969). Indeed, magnitude of illusion in a variety of figures varies systematically with changes in the angle of intersection between test and inducing contours. But, because maximum illusion occurs at different intersect angles for different figures (e.g.. 20 deg for Zöllner illusion-Wallace \& Crampin, 1969; White. 1971; and $10 \mathrm{deg}$ for Poggendorff illusion-St. Velinsky, 1925), it may be that angular distortion in the various figures follows difterent mechanisms.

Two general tindings seem to contradict the primacy of acute angle enlargement. First, Restle $(1969)$ reported a small negative illusion in a variation of the Poggendorff containing only acute angles.

This experiment was conducted at the University of Otago, New Zeatland. Jeffrey Eichengreen and Robert $H$. Cormack contributed some especially useful conments towards the revision of the manusclipt.
Restle employed the method of single stimuli in conjunction with magnitude estimation. If the method of adjustment is used, however, there is a small positive illusion (Day, 1973). Furthermore, because the oblique transversals produce a small misalignment by themselves (Day, 1973; Goldstein \& Weintraub, 1972; Green \& Hoyle, 1964), Restle's (1969) choice of the estimate corresponding to zero error (given by the estimate for alignment of single transversals) may have been underestimated. It therefore appears that the question of a small negative illusion in the Poggendorff requires further empirical examination.

Second, Coren (1970a, b) has argued that replacing the test contours by rows of dots in the Poggendorff and Zöllner figures removes the acute intersect angles and should therefore eliminate the illusion if it depends on the presence of acute angles. In dotted figures, the illusion is reduced, but not completely eliminated. White (1972), however, suggests that dotted contours in the Zöllner illusion function as low-contrast continuous contours. Thus, the angular distortion observed in figures containing dotted contours may be the result of reduced interaction between low-contrast continuous contours, as is observed in the Mueller-Lyer illusion (Wickelgren, 196.5). In fact, as the density of the dotted contours is reduced, illusion magnitude in the Zöllner display decreases (White, 1972).

In conclusion. the arguments that acute-angle versions of the Poggendorff tigure exhibit negative illusion and that acute angles are not present in dotted tigures which nevertheless manifest illusion cannot be taken as conclusive evidence against a description of illusion in terms of acute angle distortion.

Error in judging the test lines of the Zöllner illusion as parallel has been described in terms of acute angle enlargement (Wallace, 1969; White, 1971). The retinal image, rather than the perceived characteristics of the contiguration. determines the illusion (Horrell. 1971a), the illusion is tied to retinal rather 


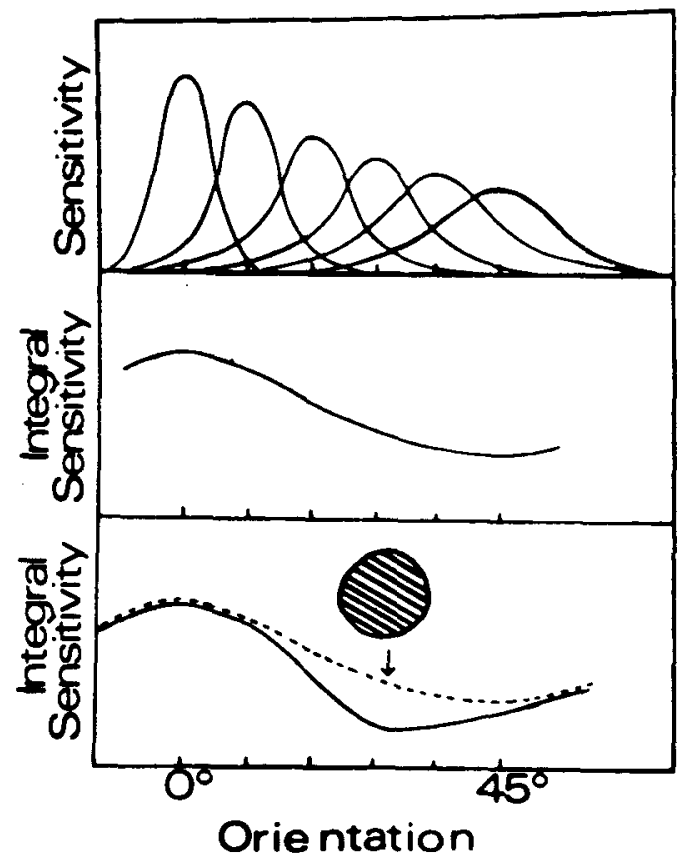

Figure 1. Sensitivity of hypothetical orientation detectors having different tuning characteristics (cf. Andrews, 1967). Middle: Integrating sensitivity of the detectors produces a sensitivity envelope (cf. Bouma \& Andriessen, 1970) which alters owing to adaptation of detectors responsive to orientations in the vicinity of the inducing contours (bottom).

than gravitational coordinates (Wallace $\&$ Moulden. $19^{-} 3$ ). and it is the angle of intersection between test and inducing contours as opposed to the overall form of the inducing contours which is important (Horrell. $19^{-} 1$ b). Specitically, the illusion is considered as the sum of individual angular distortion effects at the single intersections of the test and inducing contours; increasing the number of intersections by increasing the density of the background lines simply multiplies the overall illusion (Wallace \& Crampin. 1969; White. 19?1).

Recent neurophysiological explanations of angular distortion observed with simultaneously or successively presented intersecting lines have attributed the angular distortion or contrast effect to lateral inhibitory interaction between neural analyzers (orientation detectors) in the visual cortex which respond selectively to orientation (Andrews, 1965; Blakemore, Carpenter, \& Georgeson, 1970; Burns \& Pritchard, 1971; Howard, 1971; Over, Broerse \& Crassini, 1972; Walker, 1973; Wallace \& Moulden. 19-3). Interaction between the Hubel and Wiesel (1962. 1968) cortical orientation detectors might correspond to lateral inhibitory interaction observed at the retinal level (Ganz, 1966; Ratliff, 1965). Other explanations attribute the contrast effect to selective adaptation or response suppression of the orientation detectors (Bouma \& Andriessen. 1970; Coltheart, 19־1: Day. 1969; Over. 1971). Both lateral inhibition and adaptation explanations assume that the orientation detectors are responsive not only to a specitic "preferred" orientation. but also to a lesser extent to orientations $20-30 \mathrm{deg}$ either side of the preferred orientation (Campbell. Cleland, Cooper, \& Enroth-Cugell, 1968: Campbell \& Kulikowski. 1966: Henry. Bishop, Tupper, \&Dreher, 1973). It has further been suggested that the detectors specific to orientations either have different tuning characteristics (Andrews. 1967) or they have the same tuning characteristics but their frequency distribution along the orientation axis is not uniform (Bouma \& Andriessen, 1968, 1970). In either case, the array of orientation detectors is differentially sensitive to different contour orientations (see Figure 1); integral sensitivity over orientation defines a "sensitivity envelope" (Bouma \& Andriessen, 1970). There is, in fact. considerable psychophysical and electrophysiological evidence that sensitivity is greater to horizontal and vertical orientations than to other orientations (reviewed by Appelle, 1972).

It is possible to account for the angular distortion observed in the Zöllner illusion in terms of selective adaptation of orientation detectors which are responsive to the inducing contours. In the case of the Zölner figure with a 30 -deg intersect angle. exposure to the inducing contours results in adaptation of orientation detectors responsive to $30 \mathrm{deg}$. In addition. detectors sensitive to orientations within 20 deg either side of 30 deg adapt in an inverse proportion to the degrees of separation between 30 deg and their preferred orientation. The sensitivity envelope within the region of $30 \mathrm{deg}$ is thus altered (see Figure 1). It is assumed that perceived orientation of a line is given by the weighted average response of detectors responding to it (cf. Coltheart. $19^{-11}$. Now because some of the detectors adapted by the inducing lines are also sensitive to the 0-deg (horizontal) test lines. the weighted average response is shifted from the horizontal. The shift (error) depends on the intersect angle: a nonmonotonic variation in error with increasing intersect angle is predicted from the tuning characteristics of the detectors.

The assumption of differential sensitivity to different orientations becomes important when orientation of the complete Zölner display is considered. If the sensitivity of orientation-specitic detectors does vary along the orientation axis, then rotating the complete Zöllner display will result in detectors with different sensitivities being stimulated. It. for example. a display with 30 -deg intersect angle (like that in Figure 2) is rotated $15 \mathrm{deg}$, then detectors responsive to $15 \mathrm{deg}$ will respond to the test lines (now at 15 deg) and detectors sensitive to both 45 and 15 deg will respond to the inducing lines. In this way. the error function is expected to change as display orientation is altered. 
The present experiment measured the magnitude of the Zöllner illusion as a joint function of intersect angle and display orientation. Judd and Courten (1905) reported changes in illusion magnitude with rotation of a Zöllner figure with 45-deg intersect angle, although the shape of the error function was unclear. Wallace and Moulden (1973) compared error functions for varying intersect angles for display orientations of 0 and $30 \mathrm{deg}$. Whereas the maximum of the function for the 0 -deg orientation was at a 15-deg intersect angle, the maximum for the 30-deg orientation shifted to a $20-\mathrm{deg}$ intersect angle. In the present experiment, intersect angle varied from 5 to $45 \mathrm{deg}$ in 5-deg steps. and display orientation varied from 0 to 90 deg in 15-deg steps. Thus it was hoped to provide a description of the way in which the error functions altered with changing display orientations.

\section{METHOD}

Apparatus and stimuli were similar to those used in previous experiments (White, 1971, 1972). Nine stimulus displays were black ink drawings on white card (Figure 2). Each display comprised a series of $1-1 \mathrm{~mm}$ - $\mathrm{wide}$ inducing lines spaced $15 \mathrm{~mm}$ apart. The angle of intersection between the inducing lines and the parallel test lines was one of 5 to $45 \mathrm{deg}$ in 5 -deg steps. The circular display was $15.5 \mathrm{~cm}$ in diam. A 10th display was a blank white card without inducing lines. Two 1-mm-wide black test lines were superimposed on the background and could be varied in tilt. The test lines were 40 mm apart at their fixed left-hand end (in the horizontal figure), and adjustment of a handwheel by the subject cond effect their simultaneous convergence or divergence. Luminance of the white background hield was $41 \mathrm{~mL}$, as measured by an SEI photometer. The complete stimulus display could be rotated about a central axis (hidden from the subject) clockwise from the horizontal through 9) deg (see Figure 2). Seven values of display orientation were chosen. 0 to 9) deg in 15-deg steps.

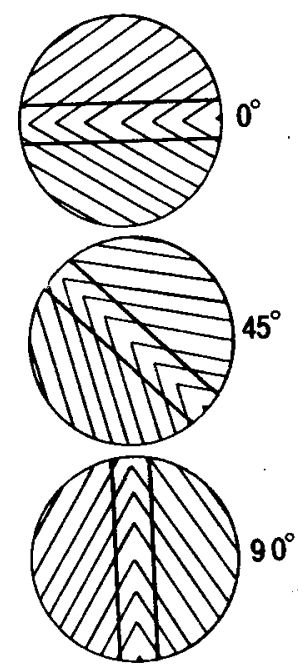

Figure 2. Zöllner displays in the present experiment. Orientations of 0,45 , and 90 deg are shown for a display with 30 -deg intersect angle. Other displays with intersect angles from 5 to $45 \mathrm{deg}$ in 5-deg steps were presented at orientations of 0 to 90 deg in 15-deg steps.

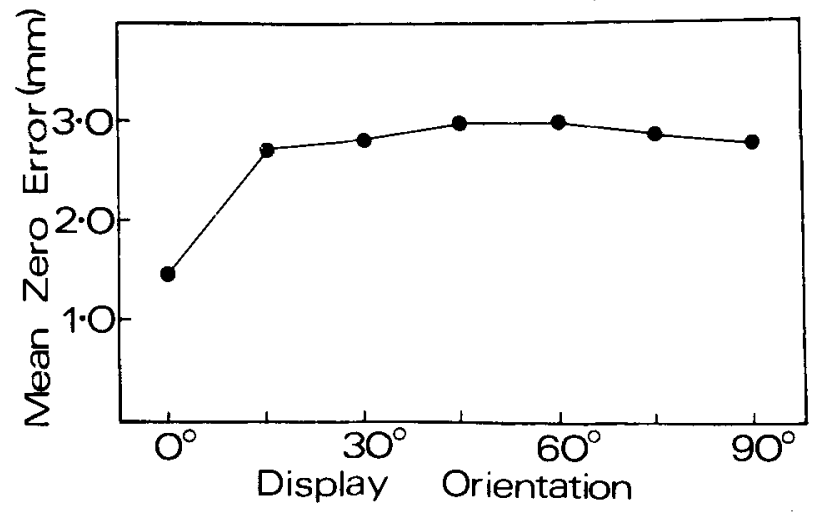

Figure 3. Estimates of bias ("zero error") in judging parallelity of test lines presented against a blank background, as a function of display orientation.

The stimulus display was placed in the frontoparallel plane at a distance of $80 \mathrm{~cm}$ from the subject. The subject viewed the display monocularly through an $18-\mathrm{cm}$-long $\times$ 6-cm-diam black cardboard reduction tube mounted in a screen which obscured the apparatus from the subject's view. The resultant stimulus field comprised the central $15.5-\mathrm{cm}$-diam display surrounded by a $4.5-\mathrm{cm}$-wide white background annulus. Thus there were no depth or orientation cues available to the subject. The subject's head was held steady in a headrest. and the experiment was conducted in a darkened room.

Three females and one male with normal or corrected-to-normal vision served as subjects. Each subject participated in seven sessions. Consecutive sessions were separated by at least $24 \mathrm{~h}$. At the beginning of a session, the subject was instructed to position the two test lines so that they looked as though they were parallel. The experimenter set the test lines to an obviously divergent position and inserted a new stimulus display before each judgment. Each adjustment required $8-10 \mathrm{sec}$. Magnitude of illusion was measured as the deviation in millimeters of the upper test line from the position of parallel at the right of the figure. A deviation of $1 \mathrm{~mm}$ corresponded to $0.35 \mathrm{deg}$ of angle between the test line and its parallel position. In any one session, display orientation was kept constant. Within a session, each of the 10 displays was presented five times, randonized over tive blocks of 10 trials in order to balance for within-session practice effects. The random orders of display orientations across sessions for two subjects were reversed for the other two subjects.

\section{RESULTS}

Mean illusion magnitudes (in millimeters, where $1 \mathrm{~mm}$ was equivalent to $0.35-\mathrm{deg}$ error) were determined for each intersect angle and display orientation separately for individual subjects. In addition, the mean estimate of parallel was obtained for each orientation of the test lines viewed against the blank white card to provide a measure of "bias." Figure 3 shows that the magnitude of bias did not change as test-line orientation was varied $[F(6,18)=$ 1.98. $\mathrm{p}>.05]$.

For each display orientation, mean illusion magnitude corrected for zero error was determined by subtracting the bias from the mean illusion for each intersect angle for individual subjects. Figure 4 presents these data averaged across subjects. The effect of intersect angle on illusion magnitude was 


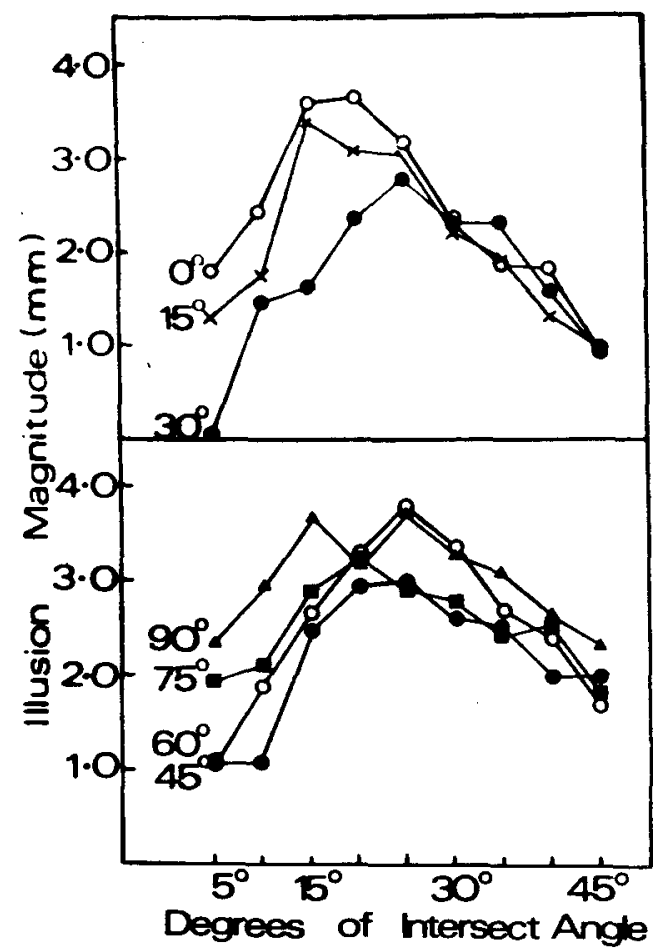

Figure 4. Illusion magnitude (corrected for bias) as a function of intersect angle (where $1 \mathrm{~mm}=0.35 \mathrm{deg}$ ). The parameter is display orientation.

highly reliable $[F(8.24)=15.22 \mathrm{p}<.001]$, although the overall effect of display orientation was not signiticant $[F(6.18)=1.07, p>.05]$. There was, however, a significant interaction between the effects of intersect angle and display orientation $[F(48,144)$ $=1.68 . \mathrm{p}<.011$.

The interaction between intersect angle and figure orientation is shown by the intersect angle at which the error functions of Figure 4 exhibit maxima. There seems to be general, although small, shift in the error functions in the direction towards greater intersect angles as display orientation is changed from 0 through $45 \mathrm{deg}$. This shift was examined further by regarding the error functions (for individual subjects) as grouped frequency distributions and computing the distribution mean (in degrees of intersect angle) for each display orientation. Figure 5 shows that as display orientation changes, the mean of the error function first increases to a maximum at $60-\mathrm{deg}$ orientation, and then decreases $[\mathrm{F}(6.18)=3.05$, $\mathrm{p}<.05]$.

\section{DISCUSSION}

The present data clearly demonstrate the characteristic variation in magnitude of the Zöllner illusion as the intersect angle between test and inducing contours increases from 5 through $45 \mathrm{deg}$. The error functions obtained for 0-deg display orientation closely correspond to previously reported functions (Wallace \&Crampin, 1969; White, 1971). In particular, maximal error is located at about 20-deg intersect angle when the display is horizontal $(0 \mathrm{deg})$. If the error is to be accounted for in terms of selective adaptation of cortical orientation detectors, then the intersect angle giving maximum error might reflect the tuning characteristics of the detectors. When the tigure is rotated so that the parallel test lines stimulate detectors responsive to orientations between 0 and $45 \mathrm{deg}$, then the intersect angle for maximum error will increase according to the increase in the tuning width of the stimulated detectors. Since detectors selective to $45 \mathrm{deg}$ are the least finely tuned (Campbell \& Kulikowski. 1966), the intersect angle for maximum error should increase with display rotation from 0 to $45 \mathrm{deg}$ and decrease with further rotation from 45 to $90 \mathrm{deg}$. The modal shift does in fact correspond to an overall error distribution shift in which the distribution mean alters with display orientation (Figure 5). The present data thus provide some degree of support for an account of the Zöllner illusion in terms of the selective adaptation of orientation detectors.

The interaction observed between the effects of intersect angle and display orientation was interpreted as a shift in the maxima of the error functions as display orientation was varied. This shift was reflected in the alteration of the mean of the error functions as display orientation varied (see Figure 5). Yet the overall change in the error functions of Figure 4 is not altogether clear. Further examination of the way in which error functions change with display orientation should consider contour interaction in a single set of inducing lines (as in the orientation illusion) rather than in contigurations comprising a double set of inducing lines (as in the Zöllner tigure of the present experiment).

On the assumption of differential tuning of the orientation detectors, lateral inhibition between

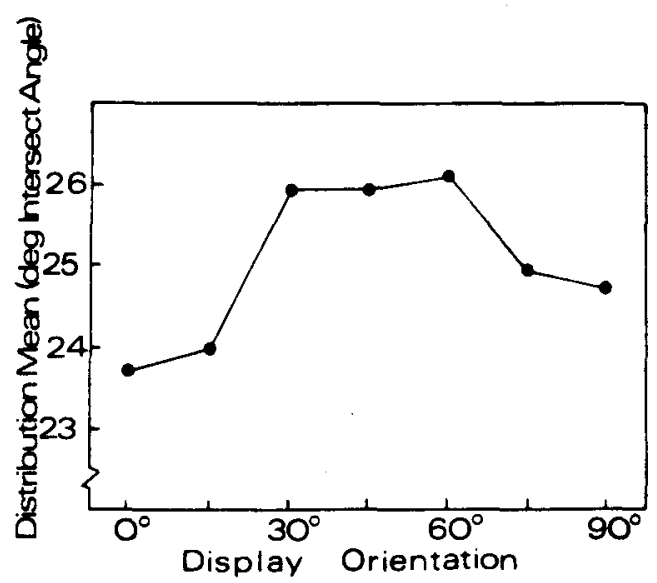

Figure 5. Means (in degrees, of intersect angle) of error distributions as a function of display orientation. 
detectors (rather than adaptation of detectors) is also able to predict modal shift of the error distribution with changing display orientation (Wallace \& Moulden, 1973). Unfortunately, the present data do not allow a choice between the adaptation and lateral inhibition explanations. The applicability of a lateral inhibition account to the present data may be limited, however, owing to the fact that the method of adjustment was used. While the subject adjusts the test lines to parallel, the independent variable of degrees of intersect angle between the test and inducing contours is also changing. The variable "degrees of intersect angle" thus describes the intersect angle only when the test lines are actually parallel. The independent variable is therefore better regarded as inducing-contour orientation. If the angular distortion is to be accounted for in terms of lateral inhibition, then it seems important that the intersect angle remain constant during each observation. The fact that intersect angle varied slightly with the subject's adjustment perhaps limits the applicability of a lateral inhibition account to the present data. Inducing-contour orientation did, however, remain constant for each observation. Since the selective-adaptation account refers to inducingcontour orientation rather than intersect angle as the important determinant of the illusion, variation in intersect angle with the subject's adjustment does not provide a problem for the adaptation account.

Perhaps the strongest evidence contraindicating lateral inhibitory interaction is that removing part of the inducing contours in various figures to eliminate acute angles while retaining obtuse angles still results in positive illusion (Day, 1973; Krantz \& Weintraub, 1973; Robinson, 1972). In particular, a small illusion is evident in the Zöllner display drawn with obtuse angles only (Hill, 1971), although the magnitude has not yet been measured. Nevertheless, intersecting contours are important for both the Poggendorff (Tong \&Weintraub, 1974) and Zöllner (Horrell, $1971 \mathrm{~b})$ effects. Illusion magnitude in these displays decreases as the intersection is degraded or removed (Krantz \& Weintraub, 1973; White; 1972).

It is perhaps misleading to describe the contrast effect as "acute angle enlargement." Rather, the important factor in contrast may be directional distortion in that the visual system inaccurately computes the direction (or orientation) of the intersecting contours (Walker, 1973). Orientationspecific adaptation of the detectors can account for directional distortion so long as the inducing and test contours stimulate proximal regions of the retina. A more complete account of the Zöllner illusion and perhaps other contrast effects observed with intersecting lines could follow explanations of orientation illusions and aftereffects in terms of adaptation of orientation-specific detectors in the cortex (Coltheart. 1971; Over, 1971).

\section{REFERENCES}

ANDREWS, D. P. Perception of contours in the central fovea. Nature, 1965, 205, 1218-1220.

ANDREws, D. P. Perception of contour orientation in the central fovea. Vision Research, 1967, 7, 975-1013.

Appelle, S. Perception and discrimination as a function of stimulus orientation: The "oblique effect" in man and animals. Psychological Bulletin, 1972, 78, 266-278.

BÉKÉsY, G. voN. Sensory inhibition. Princeton, N.J: Princeton University Press, 1967.

Berliner, A., \& Berliner, S. The distortion of straight and curved lines in geometrical fields. American Journal of Psychology, 1948, 61, 153-166.

Blakemore, C., Carpenter, R. H. S., \& Georgeson, M. A. Lateral inhibition between orientation detectors in the human visual system. Nature, 1970, 228, 37-39.

Bouma, H., \& ANDriessen, J. J. Perceived orientation of isolated line segments. Vision Research, 1968, 8, 493-507.

BOUMA, H., \& ANDRIESSEN, J. J. Induced changes in the perceived orientation of line segments. Vision Research, 1970, 10, 333-349.

Burns, B. D., \& Pritchard, R. Geometrical illusions and the response of neurones in the cat's visual cortex to angle patterns. Journal of Physiology, 1971, 213, 599-616.

Campbell, F. W., Cleland, B. G., Cooper, G. F., \& ENRoth-Cugell, C. The angular selectivity of visual cortical cells to moving gratings. Journal of Physiology, 1968, 198, 237-250.

Campbell, F. W., \& Kulikowski, J. J. Orientation selectivity of the human visual system. Journal of Physiology, 1966, $187,437-445$.

Coltheart, M. Visual feature-analyzers and aftereffects of tilt and curvature. Psychological Review, 1971, 78, 114-121.

Coren, S. Lateral inhibition and the Wundt-Hering illusion. Psychonomic Science, 1970, 18, 341. (a)

Coren, S. Lateral inhibition and geometric illusions. Quarterly Journal of Experimental Psychology, 1970, 22, 274-278. (b)

DAY, R. H. Inappropriate constancy explanation of spatial distortions. Nature, 1965, 207, 891-893.

DAY, R. H. Human perception. Sydney: Wiley, 1969.

DAY, R. H. The Poggendorff illusion with obtuse and acute angles. Perception \& Psychophysics, 1973, 14, 590-596.

Ganz, L. Mechanism of the figural aftereffects. Psychological Review, 1966, 73, 128-150.

Goldstein, M. B., \& Weintraub, D. J. The parallel-less Poggendorff: Virtual contours put the illusion down but not out. Perception \& Psychophysics, 1972, 11. 353-355.

Green, R. T., \& Hoyle, E. M. The influence of spatial orientation on the Poggendorff illusion. Acta Psychologica, 1964, 22 348-366.

Helmholtz, H. von. Handbuch der physiologischen Optik III (1866). Trans. by J. P. C. Southall (Ed.), Helmholtz's treatise on physiological optics. New York: Dover, 1962.

Henry, G. H., Bishop, P. O., Tupper, R. M., \& Dreher, B. Orientation speciticity and response variability of cells in the striate cortex. Vision Research, 1973, 13, 1771-1779.

Hering, E. Beitrage zur Physiologie. Leipzig: Engelmann, 1861.

Hill, A. L. On the enlargement of acute angles. Perceptual and Motor Skills, 1971, 33, 1238.

Horrell, R. I. Retinal image or perceived features as determinants of error in geometric illusions? Quarterly Journal of Experimental Psychology, 1971, 23, 97-106. (a)

Horrel , R. I. The angle of 'intersection of contours as the determinant of a geometric illusion. Perception \& Psychophysics, 1971, 10. 208-210. (b)

Howard, R. B. Neurophysiological models of figural aftereffects and visual illusions. Psychonomic Monograph Supplements. $1971,4,57.72$

Hubel, D. H., \& Wiesel, T. N. Receptive tields, binocular interaction and functional architecture in the cat's visual cortex. Journal of Physiology, 1962, 160, 106-154. 
HubeL, D. H., \& WIESEL. T. N. Receptive fields and functional architecture of monkey striate cortex. Joumal of Physiology, $1968,195,215-243$.

Judd, C. H., \& Courten, H. C. The Zöllner illusion. Psychological Monographs, 1905. 7. 112-139.

Krantz, D. H., \& Weintraub, D. J. Factors affecting perceived orientation of the Poggendorff transversal. Perception \& Psichophysics, 1973. 14.511-517.

OVER, R. Comparison of normalization theory and neural enhancement explanation of negative aftereffects. Psychological Bulletin, 1971, 75, 225-243.

Over. R., Broerse. J., \& Crassini, B. Orientation illusion and masking in central and peripheral vision. Joumal of Experimental Psychologiv 1972, 96. 25-31.

PIAGet, J. The mechanisms of perception. London: Routledge \& Kegan Paul, 1969.

RATLIFF, F. Mach bands: Quantitative studies on neural networks in the retina. San Francisco: Holden-Day, 1965.

Restle, F. Illusions of bent line. Perception \& Psychophysics, $1969,5,273-274$.

RoBinson, J. O. The psychology of visual illusion. London: Hutchinson, 1972.

St. Velinsky. Explication physiologique de l'illusion de Poggend orff. Année Psychologique, 1925, 26, 107.116.
Tong. L., \& Weintraub. D. J. Contour displacements and tracking errors: Probing 'twixt Poggendorff parallels. Perception \& Pspchophysics, 1974, 15, 258-268.

WALKER, E. H. A mathematical theory of optical illusions and tigural aftereffects. Perception \& Psychophysics, 1973, 13. 467.486 .

Wallace, G. K. Optical illusions. Nature, 1966, 209, 327-328.

WALlaCE, G. K. The critical distance of interaction in the Zölner illusion. Perception \& Psychophysics, 1969, 5, 261-264.

Wallace. G. K.. \& Crampin, D. J. The effect of background density on the Zölner illusion. Vision Research. 1969, 9. $167-177$.

Wallace, G. K., \& Movlden, B. The effect of body tilt on the Zollner illusion. Quarterly Journal of Experimental Psychology, $1973,25,10-21$.

White, K. G. Zöllner illusion as perceptual enlargement of acute angles. Psychonomic Science, 1971, 24, 73-75.

White, K. G. Implicit contours in the Zollner illusion. Americun Journal of Psychology, 1972. 85, 421-424.

WiCKELGREN, B. G. Brightness contrast and length perception in the Muiller-Lyer illusion. Vision Research. 1965, 5, 141-150.

(Received for publication September 16, 1974; revision received December $9,1974$. 